\title{
Vildagliptin is Effective for Glycemic Control in Diabetic Patients Undergoing either Hemodialysis or Peritoneal Dialysis
}

\author{
Hiroyuki Ito $\cdot$ Mizuo Mifune $\cdot$ Eriko Matsuyama $\cdot$ Masahide Furusho • \\ Takashi Omoto · Masahiro Shinozaki • Shinya Nishio • Shinichi Antoku • \\ Mariko Abe $\cdot$ Michiko Togane $\cdot$ Shoji Koga $\cdot$ Tsutomu Sanaka
}

To view enhanced content go to www.diabetestherapy-open.com

Received: May 5, 2013 / Published online: June 26, 2013

(c) The Author(s) 2013. This article is published with open access at Springerlink.com

\section{ABSTRACT}

Introduction: Vildagliptin can be used in patients with type 2 diabetes mellitus and renal impairment. However, there have been few reports investigating the clinical effectiveness of vildagliptin in diabetic patients undergoing hemodialysis. No previous studies have evaluated the use of vildagliptin in patients undergoing peritoneal dialysis. The authors determined the usefulness of vildagliptin for treating type 2 diabetic patients receiving chronic dialysis, including peritoneal dialysis.

H. Ito $(\varangle) \cdot$ M. Mifune - M. Furusho - T. Omoto ·

M. Shinozaki - S. Nishio - S. Antoku - M. Abe .

M. Togane

Department of Diabetes, Metabolism and Kidney

Disease, Edogawa Hospital, 2-24-18, Higashikoiwa,

Edogawa-ku, Tokyo 133-0052, Japan

e-mail: ito@edogawa.or.jp

M. Mifune - E. Matsuyama - S. Koga

Dialysis Center, Edogawa Hospital, Tokyo, Japan
Methods: A retrospective study of ten diabetic patients undergoing peritoneal dialysis and five diabetic patients undergoing hemodialysis who were treated with $50 \mathrm{mg} /$ day of vildagliptin was performed. Clinical parameters were investigated for a period of 6 months starting from the vildagliptin therapy.

Results: The hemoglobin A1c (HbA1c) levels were significantly reduced after baseline in both the peritoneal dialysis and hemodialysis groups, whereas the hemoglobin levels did not change during the follow-up period. The mean change in the HbA1c level $(\Delta \mathrm{HbA} 1 \mathrm{c})$ was $-0.6 \pm 0.9 \%$ and $-0.5 \pm 0.7 \%$ among the patients

\section{E. Matsuyama}

Department of Nursing, Edogawa Hospital, Tokyo, Japan

S. Koga

Department of Urology, Edogawa Hospital, Tokyo, Japan

T. Sanaka

Lifestyle and CKD Center, Edogawa Hospital, Tokyo, Japan 
undergoing peritoneal dialysis and hemodialysis, respectively. The glycated albumin (GA) levels were also significantly reduced compared with baseline in the peritoneal dialysis group, although the serum albumin levels did not change. The mean change in the GA level $(\Delta \mathrm{GA})$ was $-3.4 \pm 3.1 \%$ and $-2.1 \pm 2.5 \%$ among the patients undergoing peritoneal dialysis and hemodialysis, respectively. Stepwise multivariate analyses demonstrated the level of HbA1c at baseline to be significantly associated with the $\triangle \mathrm{HbA} 1 \mathrm{c}$ and that the level of GA at baseline was significantly associated with the $\Delta \mathrm{GA}$.

Conclusion: Vildagliptin exhibits effectiveness in patients with type 2 diabetes mellitus undergoing peritoneal dialysis or hemodialysis. The degree of improvement in the HbA1c and GA levels was dependent on these levels at baseline, similar to the findings of previous reports of subjects without end-stage kidney disease.

Keywords: Diabetes; End-stage kidney disease; Hemodialysis; Hemoglobin A1c; Glycated albumin; Peritoneal dialysis; Renal impairment; Type 2 diabetes mellitus; Vildagliptin

\section{INTRODUCTION}

Diabetic nephropathy is a major vascular complication in patients with type 2 diabetes mellitus. The number of individuals undergoing maintenance dialysis due to diabetic nephropathy has risen to more than 100,000 in Japan [1]. It is important to manage the blood glucose level, even in diabetic patients undergoing maintenance dialysis, because good glycemic control prevents the development of diabetic macroangiopathy and improves prognosis [2-5]. Hyperglycemia in patients with renal impairment is difficult to control due to limited therapeutic options and the high prevalence of diabetic complications. Although insulin injection is a basic therapy in patients with hyperglycemia and renal dysfunction, visual disturbances caused by diabetic retinopathy, hemiplegia secondary to cerebrovascular disease, and/or dementia, which is frequently observed in patients with diabetes [6], are factors that limit the continuation of insulin therapy. When oral hypoglycemic agents (OHAs) are prescribed for diabetic patients with renal insufficiency, sulfonylureas, biguanides, and thiazolidines should be used carefully due to side effects. Therefore, alpha-glucosidase inhibitors and glinides are used in such patients. However, glycemic control is often insufficient in diabetic patients with chronic renal failure as insulin resistance is commonly observed [7].

Vildagliptin, a dipeptidyl peptidase (DPP)-4 inhibitor, is a novel OHA that can be used in patients with type 2 diabetes mellitus and renal impairment [8-10]. However, few reports have investigated the clinical effectiveness of vildagliptin in diabetic patients undergoing hemodialysis [11, 12]. Furthermore, no previous studies have evaluated the use of vildagliptin in patients undergoing peritoneal dialysis.

In the present study, the authors determined the clinical usefulness of vildagliptin for treating type 2 diabetic patients receiving chronic dialysis, including peritoneal dialysis.

\section{MATERIALS AND METHODS}

\section{Ethics Statement}

This study was conducted according to the principles expressed in the 2008 Declaration of 
Helsinki. The Ethics Committee of Edogawa Hospital approved the protocol of this study and waived the need for written informed consent because the data were analyzed anonymously for this study based on the information stored in the hospital database.

\section{Patients and Methods}

Subjects with type 2 diabetes mellitus from patients with end-stage kidney disease undergoing chronic dialysis at the Dialysis Center of Edogawa Hospital, Tokyo, Japan in February 2013 were eligible for this study. Nondiabetic subjects and patients treated with insulin or OHAs other than vildagliptin were excluded. The clinical parameters were investigated for 6 months starting from baseline (the initial date of vildagliptin therapy). The indication for vildagliptin therapy was determined by the attending physicians. Blood samples were collected before dialysis in the patients undergoing hemodialysis and at hospital visit after breakfast in the patients undergoing peritoneal dialysis.

The hemoglobin A1c (HbA1c) and glycated albumin (GA) levels were determined according to a high performance liquid chromatography method using an automated HLC-723G9 analyzer (Tosoh Corporation, Tokyo, Japan) and an enzymatic method (Lucica ${ }^{\circledR}$ GA-L, Asahi Kasei Pharma Corporation, Tokyo, Japan) on a biochemical autoanalyzer, respectively.

\section{Statistical Analysis}

All data are presented as the mean $\pm \mathrm{SD}$. Analyses of variance (ANOVA) were used for between-group comparisons of continuous variables. The paired $t$ test was employed to determine whether there were any differences in the levels of blood glucose, HbA1c, GA, hemoglobin, or serum albumin during the follow-up period compared with the baseline values. Pearson's univariate regression analyses and stepwise multivariate analyses using the forward selection method were performed to determine the associations between the changes in the HbA1c level $(\triangle \mathrm{HbA} 1 \mathrm{c})$ or the GA level $(\Delta \mathrm{GA})$ and the other clinical parameters. When clinical data were missing, the appropriate value obtained on the previous visit was used according to the last observational carried forward (LOCF) method. Differences of $P<0.05$ (two-tailed) were considered to be statistically significant. The statistical software package JMP, version 8.0 (SAS Institute, Cary, NC, USA), was used to perform all the analyses.

\section{RESULTS}

\section{Patients Demographics and Baseline Characteristics}

Fifty-nine subjects with type 2 diabetes mellitus from 97 patients undergoing chronic dialysis were eligible for this study. Thirty-eight nondiabetic subjects and patients treated with insulin $(n=16)$, liraglutide $(n=2)$, pioglitazone $(n=1)$, alogliptin $(n=1)$, and mitiglinide $(n=1)$ among 59 subjects with type 2 diabetes mellitus were excluded. Finally, a retrospective study of ten patients receiving peritoneal dialysis [six subjects undergoing continuous ambulatory peritoneal dialysis (CAPD) and four subjects undergoing continuous cycler peritoneal dialysis (CCPD) using a $1.5 \%$ glucose solution and/or icodextrin as the dialysis solution] and five patients receiving hemodialysis who were treated with $50 \mathrm{mg} /$ day of vildagliptin was performed. Twenty-three 
diabetic patients without any pharmacological treatment for hyperglycemia [18 subjects on peritoneal dialysis (eight CAPD and ten CCPD patients using 1.5\% glucose and/or icodextrin) and five subjects on hemodialysis] were also investigated as control subjects. Treatment with vildagliptin was initiated with a change from combination therapy consisting of $150 \mathrm{mg} /$ day of miglitol and $1 \mathrm{mg} /$ day of glimepiride in one patient undergoing peritoneal dialysis and one patient undergoing hemodialysis in place of insulin treatment according to a sliding scale administration. The remaining 13 patients in the vildagliptin group received no pharmacological therapy before the initiation of vildagliptin.

Table 1 shows the baseline characteristics of the study subjects. The duration of dialysis was significantly longer in the vildagliptin group than in the control group. All subjects were treated with vildagliptin monotherapy for glycemic control during the follow-up period in the vildagliptin group.

\section{Efficacy}

In the vildagliptin group, the blood glucose levels did not change significantly during the follow-up period (Table 2). The HbA1c levels were significantly reduced after the initiation of vildagliptin therapy in both the hemodialysis and peritoneal dialysis groups, whereas the hemoglobin levels did not change during the follow-up period. The mean $\Delta \mathrm{HbA} 1 \mathrm{c}$ was $-0.6 \pm 0.9 \%$ and $-0.5 \pm 0.7 \%$ among the patients undergoing peritoneal dialysis and hemodialysis, respectively. The GA levels were also significantly reduced compared with baseline in the peritoneal dialysis group, although the serum albumin levels did not change. The mean $\Delta \mathrm{GA}$ was $-3.4 \pm 3.1 \%$ and $-2.1 \pm 2.5 \%$ among the patients undergoing peritoneal dialysis and hemodialysis, respectively. The blood glucose, HbA1c, and GA levels at the baseline were higher in the vildagliptin group than in the control group. The blood glucose and HbA1c levels increased

Table 1 Baseline characteristics of the patients

\begin{tabular}{|c|c|c|c|c|}
\hline & \multicolumn{2}{|l|}{ Vildagliptin group } & \multicolumn{2}{|l|}{ Control group } \\
\hline & $\begin{array}{l}\text { Peritoneal dialysis } \\
(n=10)\end{array}$ & $\begin{array}{l}\text { Hemodialysis } \\
(n=5)\end{array}$ & $\begin{array}{l}\text { Peritoneal dialysis } \\
(n=18)\end{array}$ & $\begin{array}{l}\text { Hemodialysis } \\
(n=5)\end{array}$ \\
\hline Age (years) & $66 \pm 8$ & $65 \pm 8$ & $62 \pm 12$ & $63 \pm 14$ \\
\hline Male (\%) & 100 & 100 & 89 & 100 \\
\hline Duration of diabetes mellitus (years) & $14 \pm 10$ & $20 \pm 11$ & $10 \pm 9$ & $16 \pm 13$ \\
\hline Duration of dialysis (months) & $8 \pm 8^{\$}$ & $15 \pm 10^{\$ \$}$ & $19 \pm 10$ & $53 \pm 30$ \\
\hline Serum total protein $(\mathrm{g} / \mathrm{dL})$ & $6.1 \pm 0.5$ & $6.4 \pm 0.5$ & $6.4 \pm 0.5$ & $7.1 \pm 0.4$ \\
\hline Aspartate transaminase (IU/L) & $17 \pm 4$ & $39 \pm 49$ & $20 \pm 10$ & $15 \pm 3$ \\
\hline Alanine Transaminase (IU/L) & $15 \pm 5$ & $23 \pm 30$ & $15 \pm 12$ & $10 \pm 5$ \\
\hline Serum creatinine $(\mathrm{mg} / \mathrm{dL})$ & $6.5 \pm 0.9$ & $7.5 \pm 1.9$ & $8.0 \pm 3.6$ & $10.3 \pm 3.0$ \\
\hline Blood urea nitrogen $(\mathrm{mg} / \mathrm{dL})$ & $47 \pm 15$ & $53 \pm 16$ & $47 \pm 17$ & $54 \pm 7$ \\
\hline Serum uric acid (mg/dL) & $6.7 \pm 1.4$ & $6.3 \pm 1.3$ & $7.0 \pm 2.0$ & $6.3 \pm 0.9$ \\
\hline
\end{tabular}

$\$ P<0.05$ vs. control group, ${ }^{\$} P<0.01$ vs. control group 
Table 2 Changes in the clinical parameters during the observation period

\begin{tabular}{|c|c|c|c|c|c|c|c|c|}
\hline & \multicolumn{4}{|c|}{ Vildagliptin group } & \multicolumn{4}{|c|}{ Control group } \\
\hline & Baseline & 1 month & 3 months & 6 months & Baseline & 1 month & 3 months & 6 months \\
\hline \multicolumn{9}{|c|}{ Blood glucose $(\mathrm{mg} / \mathrm{dL})$} \\
\hline Peritoneal dialysis & $167 \pm 62^{\$}$ & $145 \pm 52$ & $160 \pm 31$ & $158 \pm 31$ & $126 \pm 37$ & $137 \pm 33$ & $147 \pm 44$ & $151 \pm 38^{\#}$ \\
\hline Hemodialysis & $160 \pm 38$ & $151 \pm 38$ & $138 \pm 30$ & $132 \pm 29$ & $137 \pm 55$ & $163 \pm 87$ & $144 \pm 49$ & $159 \pm 94$ \\
\hline \multicolumn{9}{|l|}{ HbAlc (\%) } \\
\hline Peritoneal dialysis & $6.8 \pm 1.1^{\$}$ & $6.5 \pm 1.2^{\# \#}$ & $6.4 \pm 1.0$ & $6.5 \pm 0.7$ & $5.9 \pm 0.7$ & $6.2 \pm 0.5^{\# \#}$ & $6.3 \pm 0.4$ & $6.5 \pm 0.5^{\#}$ \\
\hline Hemodialysis & $6.0 \pm 0.3$ & $5.7 \pm 0.2^{\#}$ & $5.6 \pm 0.3$ & $5.5 \pm 0.6$ & $5.4 \pm 0.7$ & $5.5 \pm 0.8$ & $5.6 \pm 0.8$ & $5.7 \pm 1.0$ \\
\hline \multicolumn{9}{|l|}{ GA (\%) } \\
\hline Peritoneal dialysis & $19.6 \pm 5.5$ & $19.2 \pm 5.7$ & $17.4 \pm 4.0^{\#}$ & $17.1 \pm 3.7^{\#}$ & $17.8 \pm 1.8$ & $17.5 \pm 1.5$ & $19.1 \pm 1.9$ & $17.4 \pm 0.3$ \\
\hline Hemodialysis & $21.8 \pm 2.6^{\$}$ & $20.7 \pm 3.6$ & $19.9 \pm 3.4$ & $19.7 \pm 3.3$ & $18.3 \pm 1.3$ & $17.6 \pm 2.3$ & $16.2 \pm 2.0^{\#}$ & $17.2 \pm 3.2$ \\
\hline \multicolumn{9}{|l|}{ Hemoglobin $(\mathrm{g} / \mathrm{dL})$} \\
\hline Peritoneal dialysis & $10.2 \pm 1.4$ & $10.7 \pm 1.8$ & $10.8 \pm 2.4$ & $9.7 \pm 1.8$ & $10.8 \pm 1.4$ & $11.0 \pm 1.6$ & $10.9 \pm 1.7$ & $10.8 \pm 1.3$ \\
\hline Hemodialysis & $10.3 \pm 0.5$ & $10.8 \pm 1.1$ & $11.3 \pm 1.6$ & $10.6 \pm 0.8$ & $10.1 \pm 0.6$ & $11.3 \pm 0.6^{\#}$ & $10.4 \pm 0.8$ & $9.9 \pm 0.8$ \\
\hline \multicolumn{9}{|l|}{ Serum albumin $(\mathrm{g} / \mathrm{dL})$} \\
\hline Peritoneal dialysis & $3.2 \pm 0.3$ & $3.2 \pm 0.4$ & $3.2 \pm 0.5$ & $3.1 \pm 0.5$ & $3.3 \pm 0.5$ & $3.3 \pm 0.5$ & $3.2 \pm 0.4$ & $3.1 \pm 0.5$ \\
\hline Hemodialysis & $3.7 \pm 0.4$ & $3.5 \pm 0.2$ & $3.5 \pm 0.1$ & $3.5 \pm 0.2$ & $3.9 \pm 0.5$ & $3.7 \pm 0.3$ & $3.7 \pm 0.2$ & $3.7 \pm 0.2$ \\
\hline
\end{tabular}

$H b A 1 c$ hemoglobin A1c, $G A$ glycated albumin

$\$ P<0.05$ vs. control group

${ }^{\#} P<0.05$ vs. the baseline value and ${ }^{\# \#} P<0.01$ vs. the baseline value

with the passage of time during follow-up in the control group.

Among the vildagliptin-treated patients undergoing peritoneal dialysis, the $\Delta \mathrm{HbA} 1 \mathrm{c}$ exhibited a significant negative correlation with the HbA1c level at baseline (Fig. 1), although it was not related to age, the duration of diabetes mellitus, the duration of dialysis, or the levels of hemoglobin, serum total protein, albumin, aspartate transaminase, alanine transaminase, creatinine, blood urea nitrogen, uric acid, or blood glucose. There were also significant negative correlations between the $\triangle \mathrm{GA}$ and the levels of HbA1c and GA at baseline (Fig. 2). The $\Delta \mathrm{GA}$ was not related to any of the other clinical parameters, including the blood glucose level. A stepwise multivariate analysis demonstrated that the level of HbA1c was significantly associated with the $\Delta$ HbA1c $\left(F=7.1, R^{2}=0.54, P=0.04\right)$ when the HbA1c and GA levels at baseline were defined as the independent variables. Furthermore, the level of GA tended to be associated with the $\Delta \mathrm{GA}\left(F=4.1, R^{2}=0.62\right.$, $P=0.099)$ when the HbA1c and GA levels at baseline were defined as the independent variables.

\section{DISCUSSION}

This study demonstrated that vildagliptin is clinically effective for achieving glycemic control in patients with type 2 diabetes mellitus undergoing either peritoneal dialysis or hemodialysis. There are no investigations of the clinical usefulness of vildagliptin in diabetic patients undergoing peritoneal dialysis, whereas several studies have already been reported in those undergoing hemodialysis [11-13]. Kume et al. [11] reported that the daily administration 


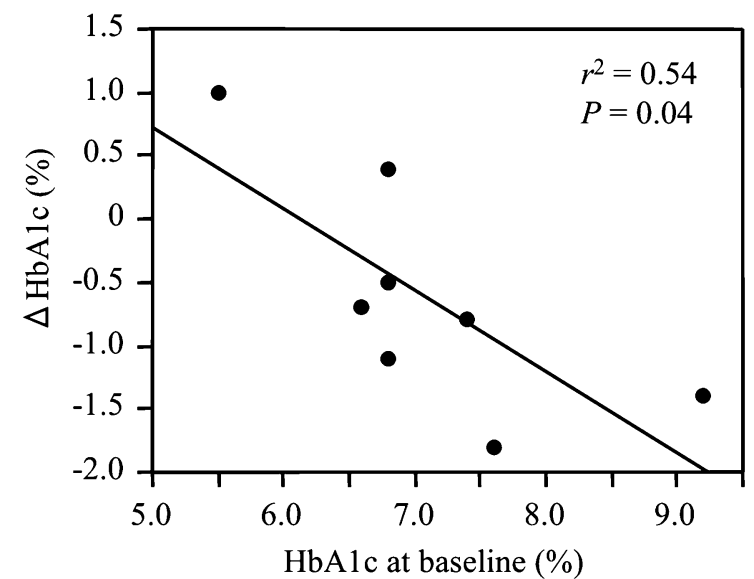

Fig. 1 Relationships between the changes in the HbAlc level $(\triangle \mathrm{HbA} 1 \mathrm{c})$ following the administration of vildagliptin and the levels of HbAlc and GA at baseline in the type 2

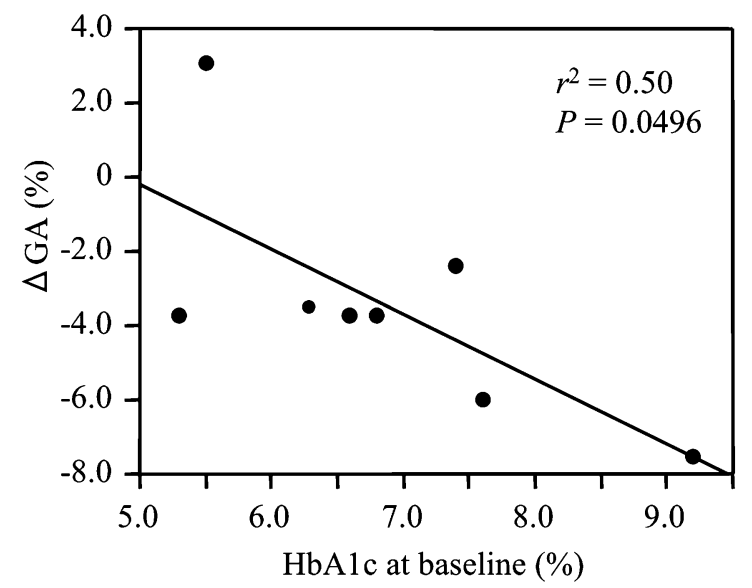

Fig. 2 Relationships between the changes in the GA level $(\triangle \mathrm{GA})$ following the administration of vildagliptin and the levels of HbAlc and GA at baseline in the type 2 diabetic

of $50 \mathrm{mg}$ of vildagliptin for 24 weeks was associated with a 2.6-point reduction in the levels of GA (23.8-21.2\%) among 23 patients with type 2 diabetes undergoing hemodialysis. Ito et al. [12] also demonstrated that the administration of vildagliptin at a dose of $50 \mathrm{mg} /$ day for 24 weeks achieved a 0.47-point reduction in the levels of $\mathrm{HbA1c}$ from $6.5 \%$ and a 3.2-point reduction in the levels of GA from $22.3 \%$ among 11 subjects with type 2 diabetes

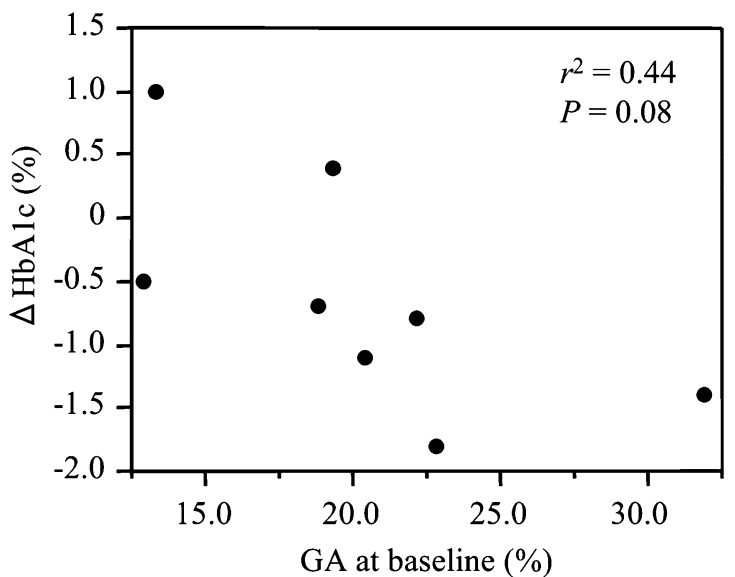

diabetic patients undergoing peritoneal dialysis. $H b A 1 c$ hemoglobin A1c, $G A$ glycated albumin

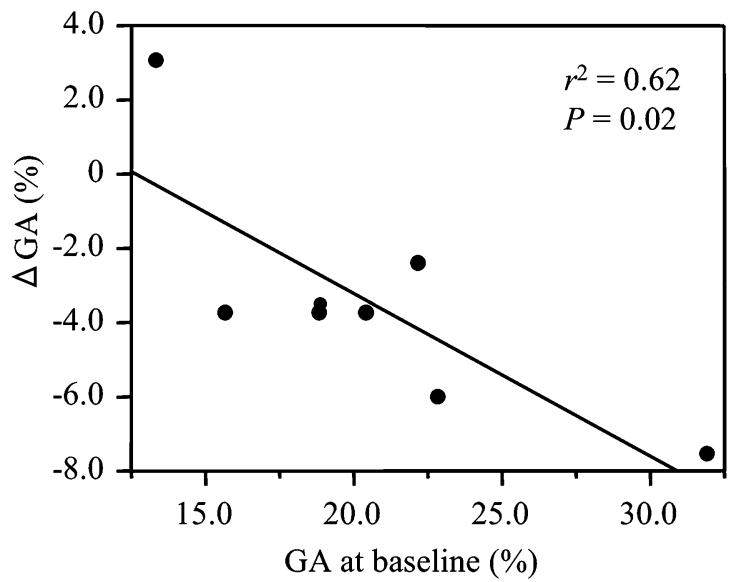

patients undergoing peritoneal dialysis. $H b A 1 c$ hemoglobin A1c, $G A$ glycated albumin

undergoing hemodialysis out of 30 patients administered $50-100 \mathrm{mg}$ of daily vildagliptin. Furthermore, Terawaki et al. [13] showed that the daily administration of $50 \mathrm{mg}$ of vildagliptin for a few days shortened the duration of hyperglycemia in ten diabetic patients undergoing hemodialysis, similar to the results achieved with the daily administration of $0.3 \mathrm{mg}$ of liraglutide, a glucagon-like peptide (GLP)-1 receptor agonist. 
It is difficult to simply compare these results due to the different basal values of hemoglobin and HbA1c; however, the reductions in the HbA1c levels observed in the present study and in the investigations of subjects undergoing hemodialysis cited above [11, 12] are smaller than those reported in diabetic patients without renal dysfunction [14-16]. The differences in the reduction of $\mathrm{HbA} 1 \mathrm{c}$ between patients with and without renal impairment may be due to the shortened turnover of red blood cells in individuals with renal insufficiency, in addition to the basal value of HbA1c.

In the present study, the $\triangle \mathrm{HbA} 1 \mathrm{c}$ and $\Delta \mathrm{GA}$ following the administration of vildagliptin were associated with the basal values of HbA1c and GA, respectively. Similarly, it has been reported that the hypoglycemic effects of DPP-4 inhibitors are dependent on the glycemic control observed at baseline in diabetic patients without renal dysfunction $[15,16]$.

The GA level has been described as being more useful as an index of glycemic control than the HbA1c level in diabetic subjects undergoing hemodialysis [17-21]. Because hypoalbuminemia is commonly detected in patients receiving peritoneal dialysis, it is difficult to determine the degree of control of blood glucose based on the GA level in patients with diabetes receiving peritoneal dialysis. However, it has been reported that the HbA1c level significantly underestimates glycemic control relative to the GA level in both hemodialysis and peritoneal dialysis patients [22]. Although the effects of vildagliptin on the GA level have not been previously investigated, both the HbA1c and GA levels were reduced by the administration of vildagliptin in the present study.

Insulin injection is an essential therapy in patients with diabetes and renal dysfunction, based on the various side effects of OHAs. The percentage of patients receiving insulin therapy usually increases in association with progression in the stage of chronic kidney disease. However, factors limiting the use of insulin therapy, such as visual disturbances, hemiplegia, and dementia, are frequently observed in subjects with end-stage kidney disease and diabetes mellitus. Because insulin excretion into the urine from the kidneys is reduced, the proportion of diabetic patients requiring pharmacological treatments among those undergoing maintenance dialysis often decreases. In the present study, the proportion of subjects receiving insulin therapy was $27 \%$ of the total number of diabetic patients; however, a comparable number of subjects required OHA treatment. DPP-4 inhibitors, including vildagliptin, are potentially powerful therapeutic agents in patients with hyperglycemia undergoing maintenance dialysis, independent from the dialysis method used.

The present study is associated with limitations that should be kept in mind when interpreting the results. First, the present study evaluated preliminary data in a short-term observation of a small number of patients. Second, the study investigated changes in glycemic control only. It is necessary to determine which vascular events are prevented by the administration of vildagliptin in a larger number of patients during a longer follow-up period.

\section{CONCLUSION}

Vildagliptin exhibits effectiveness in achieving glycemic control in patients with type 2 diabetes mellitus undergoing either peritoneal dialysis or hemodialysis. In the present study, 
the degree of improvement in the HbA1c and GA levels was dependent on these levels at baseline, similar to the findings of previous reports of subjects without end-stage kidney disease.

\section{ACKNOWLEDGMENTS}

No funding or sponsorship was received for this study or publication of this article. The authors thank Ms. Tomoko Koyanagi of the secretarial section of Edogawa Hospital for her valuable help in the data collection. Dr Ito is the guarantor for this article, and takes responsibility for the integrity of the work as a whole.

Conflict of interest. Hiroyuki Ito, Mizuo Mifune, Eriko Matsuyama, Masahide Furusho, Takashi Omoto, Masahiro Shinozaki, Shinya Nishio, Shinichi Antoku, Mariko Abe, Michiko Togane, Shoji Koga, and Tsutomu Sanaka declare no conflict of interest.

Compliance with ethics guidelines. This study was conducted according to the principles expressed in the 2008 Declaration of Helsinki. The Ethics Committee of Edogawa Hospital approved the protocol of this study and waived the need for written informed consent because the data were analyzed anonymously for this study based on the information stored in the hospital database.

Open Access. This article is distributed under the terms of the Creative Commons Attribution Noncommercial License which permits any noncommercial use, distribution, and reproduction in any medium, provided the original author(s) and the source are credited.

\section{REFERENCES}

1. Nakai S, Iseki K, Itami N, et al. An overview of regular dialysis treatment in Japan (as of 31 December 2010). Ther Apher Dial. 2012;16: 483-521.

2. Morioka T, Emoto M, Tabata $\mathrm{T}$, et al. Glycemic control is a predictor of survival for diabetic patients on hemodialysis. Diabetes Care. 2001;24:909-13.

3. Hayashino Y, Fukuhara S, Akiba T, et al. Diabetes, glycaemic control and mortality risk in patients on haemodialysis: the Japan Dialysis Outcomes and Practice Pattern Study. Diabetologia. 2007;50:1170-7.

4. Oomichi T, Emoto M, Tabata T, et al. Impact of glycemic control on survival of diabetic patients on chronic regular hemodialysis: a 7-year observational study. Diabetes Care. 2006;29: 1496-500.

5. Shima K, Komatsu M, Kawahara K, Minaguchi J, Kawashima S. Stringent glycaemic control prolongs survival in diabetic patients with end-stage renal disease on haemodialysis. Nephrology. 2010;15: 632-8.

6. Kawamura $\mathrm{T}$, Umemura $\mathrm{T}$, Hotta N. Cognitive impairment in diabetic patients: can diabetic control prevent cognitive decline? J Diabetes Invest. 2012;3:413-23.

7. Shinohara K, Shoji $T$, Emoto $M$, et al. Insulin resistance as an independent predictor of cardiovascular mortality in patients with end-stage renal disease. J Am Soc Nephrol. 2002;13:1894-900.

8. Lukashevich V, Schweizer A, Shao Q, Groop PH, Kothny W. Safety and efficacy of vildagliptin versus placebo in patients with type 2 diabetes and moderate or severe renal impairment: a prospective 24-week randomized placebocontrolled trial. Diabetes Obes Metab. 2011;13: 947-54.

9. Kothny W, Shao Q, Groop PH, Lukashevich V. Oneyear safety, tolerability and efficacy of vildagliptin in patients with type 2 diabetes and moderate or severe renal impairment. Diabetes Obes Metab. 2012;14:1032-9.

10. Lukashevich V, Schweizer A, Foley JE, Dickinson S, Groop PH, Kothny W. Efficacy of vildagliptin in combination with insulin in patients with type 2 diabetes and severe renal impairment. Vasc Health Risk Manag. 2013;9:21-8. 
11. Kume S, Uzu T, Takagi C, et al. Efficacy and tolerability of vildagliptin in type 2 diabetic patients on hemodialysis. $\mathrm{J}$ Diabetes Invest. 2012;3:298-301.

12. Ito $M$, Abe $M$, Okada $K$, et al. The dipeptidyl peptidase-4 (DPP-4) inhibitor vildagliptin improves glycemic control in type 2 diabetic patients undergoing hemodialysis. Endocr J. 2011;58: 979-87.

13. Terawaki $Y$, Nomiyama $T$, Akehi $Y$, et al. The efficacy of incretin therapy in patients with type 2 diabetes undergoing hemodialysis. Diabetol Metab Syndr. 2013;5:10.

14. Pi-Sunyer FX, Schweizer A, Mills D, Dejager S. Efficacy and tolerability of vildagliptin monotherapy in drug-naïve patients with type 2 diabetes. Diabetes Res Clin Pract. 2007;76:132-8.

15. Dejager S, Razac S, Foley JE, Schweizer A. Vildagliptin in drug-naïve patients with type 2 diabetes: a 24-week, double-blind, randomized, placebo-controlled, multiple-dose study. Horm Metab Res. 2007;39:218-23.

16. Dejager S, Schweizer A, Foley JE. Evidence to support the use of vildagliptin monotherapy in the treatment of type 2 diabetes mellitus. Vasc Health Risk Manag. 2012;8:339-48.

17. Kumeda $Y$, Inaba $M$, Shoji $S$, et al. Significant correlation of glycated albumin, but not glycated haemoglobin, with arterial stiffening in haemodialysis patients with type 2 diabetes. Clin Endocrinol. 2008;69:556-61.

18. Okada T, Nakao T, Matsumoto H, et al. Association between markers of glycemic control, cardiovascular complications and survival in type 2 diabetic patients with end-stage renal disease. Intern Med. 2007;46:807-14.

19. Fukuoka K, Nakao K, Morimoto H, et al. Glycated albumin levels predict long-term survival in diabetic patients undergoing haemodialysis. Nephrology. 2008;13:278-83.

20. Peacock TP, Shihabi ZK, Bleyer AJ, et al. Comparison of glycated albumin and hemoglobin A(1c) levels in diabetic subjects on hemodialysis. Kidney Int. 2008;73:1062-8.

21. Inaba $\mathrm{M}$, Okuno $\mathrm{S}$, Kumeda $\mathrm{Y}$, Osaka CKD Expert Research, et al. Glycated albumin is a better glycemic indicator than glycated hemoglobin values in hemodialysis patients with diabetes: effect of anemia and erythropoietin injection. J Am Soc Nephrol. 2007;18:896-903.

22. Freedman BI, Shenoy RN, Planer JA, et al. Comparison of glycated albumin and hemoglobin A1c concentrations in diabetic subjects on peritonealand hemodialysis. Perit Dial Int. 2010;30:72-9. 